\title{
Cervicothoracic Fixation by Domino Connector in Cervicothoracic Junction Tuberculosis-A Case Report
} \author{
Mohammad Shahnawaz Bari \\ ${ }^{1}$ Department of Spinal Neurosurgery, Bangabandhu Sheikh Mujib \\ Medical University, Dhaka, Bangladesh \\ ${ }^{2}$ Department of Neurosurgery, Bangabandhu Sheikh Mujib Medical \\ University, Dhaka, Bangladesh \\ ${ }^{3}$ Department of Neurosurgery, National Institute of Neurosciences, \\ Dhaka, Bangladesh
}

Md. Rezaul Amin ${ }^{1}$ Moududul Haque ${ }^{2}$ Haradhan Dev Nath ${ }^{2}$ ABM Manwar Hossain ${ }^{3}$

\begin{abstract}
Address for correspondence Md. Rezaul Amin, MBBS, MS, Department of Spinal Neurosurgery, Bangabandhu Sheikh Mujib Medical University, Dhaka, Bangladesh (e-mail: rezaul_amin@yahoo.com).
\end{abstract}

Indian J Neurotrauma:2020;17:146-148

\begin{abstract}
Keywords

- cervicothoracic

- dorsal

- medical research council

- magnetic resonance imaging

- tuberculosis

Cervicothoracic junction tuberculosis involves the C7 to D3 levels of the spine which constitutes $5 \%$ of all spinal tuberculosis. As the cervicothoracic junction is transitional zone and a weight-bearing area, the anatomical considerations for treatment of cervicothoracic junction is very difficult. We did a lateral mass screw for $\mathrm{C} 5,6$, and 7 and pedicle screw of D3 and 4 levels, and connected cervical $3.5 \mathrm{~mm}$ rod with thoracic $5.5 \mathrm{~mm}$ rod using a domino connector. We gave antituberculosis therapy for 18 months. We followed-up the patient routinely and she significantly improved.
\end{abstract}

\section{Introduction}

Cervicothoracic spinal tuberculosis involves the $\mathrm{C} 7$ to $\mathrm{D} 3$ levels of the spine, which constitutes $5 \%$ of all spinal tuberculosis. ${ }^{1,2}$ Because the cervicothoracic junction is a transitional zone and weight-bearing area, tuberculosis of this region frequently results in spinal instability, severe kyphotic deformity, and can cause severe neurological deficit.,4 Due to the anatomical location, surgical treatment of this location is very challenging. ${ }^{5}$ For this region, various approaches have been described. It remains controversial which approach is perfect for cervicothoracic region. We described spinal tuberculosis as involving D1/D2 with paravertebral collection and kyphotic deformity with paraparesis. We did single stage posterior debridement, fusion and cervicothoracic instrumentation, and connected the cervical $3.5 \mathrm{~mm}$ rod with $5.5 \mathrm{~mm}$ thoracic rod with the help of a domino connector. To my knowledge, this is the first case done in Bangladesh through this technique.

\section{Case Report}

\section{History and Presentation}

A 22-year-old female nurse came with the complaint of upper back and neck pain from the last 3 months, which gradually increased in intensity. She then developed progressive paraparesis and was unable to walk within 6 weeks from the time symptoms developed.

\section{Physical Examination}

Her lower limb power was MRC grade 3 on the right side and MRC grade 4 on the left side. Ankle and knee jerk were exaggerated and planter was extensor. She had a sensory level at D4. Her bladder and bowel functions were normal. She had occasional low-grade fever which subsided spontaneously.

\section{Imaging Studies}

MRI showed an extradural mass at the level of D1/D2 with significant cord compression and destruction of the D1/D2 vertebra with significant paravertebral collection. We planned to do a single stage posterior debridement, fusion and cervicothoracic instrumentation, by connecting the cervical $3.5 \mathrm{~mm}$ rod with $5.5 \mathrm{~mm}$ thoracic rod using a domino connector (-Fig. 1).

\section{Operation Procedure}

A surgery was planned for posterior focus debridement, fusion and cervico-thoracic instrumentation between cervical and thoracic vertebra, connected by a domino connector. 

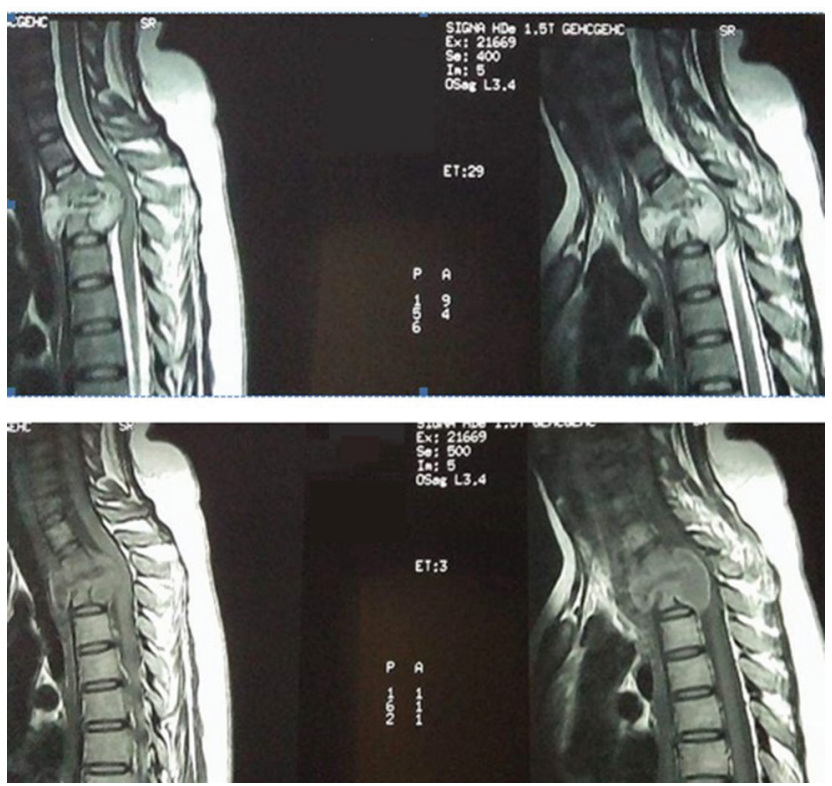

Fig. $1 \mathrm{MRI}$ of cervicothoracic spine showing destruction of D1/D2 vertebra with paravertebral collection.

So far, this is the first case done in Bangladesh through this technique.

Patient was positioned in prone with Sugita head fixator. Posterior midline incision was given, extending from C4 to D4 vertebra. After retracting the paravertebral muscles, space was identified clinically and radiologically. After laminectomy of D2 and partially D1, an extradural mass placed anterior to the cord with huge collection of pus from extradural and paravertebral was noted. Meticulous excision of the mass and drainage of the pus was done properly. Lateral mass screw was given at C5, 6 and 7 vertebra, and pedicle screw was given at D3 and 4 levels which was confirmed radiologically. A $3.5 \mathrm{~mm}$ rod was placed at cervical level and $5.5 \mathrm{~mm}$ rod was placed at dorsal level, which was connected by a domino connector. Proper construction of cervicothoracic curvature was maintained ( - Fig. 2 ).

\section{Postoperative Course}

The postoperative course was uneventful. Her neck pain improved and she could walk without support within 1 month after surgery. Histology shows granulomatous lesion and gene expert.

The result detected tuberculosis. An 18-month antituberculous therapy was planned for her. During the first 3 months, she got the 4-drug regimen and for the rest of the 15 months, 2-drug regimen was given. Pyridoxine was given throughout the treatment in anti-TB therapy (-Figs. $\mathbf{2}$ and $\mathbf{3}$ ).

\section{Follow Up}

She was advised for follow-up after 1 month of surgery. Her blood picture became normal within 1 month. The patient could walk without support and was pain-free within 1 month after surgery. The patient was advised for follow-up in every 3 months.

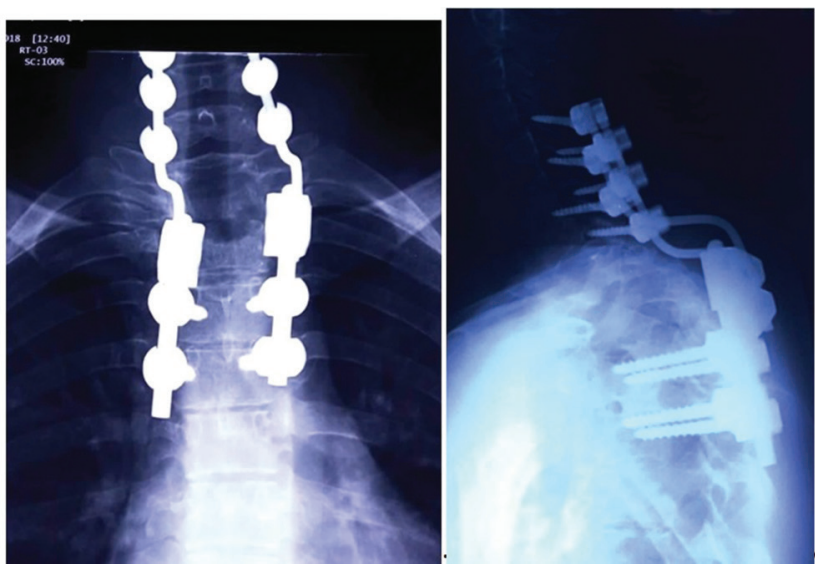

Fig. 2 Postoperative picture of cervicothoracic junction using domino connector.

\section{Discussion}

Cervicothoracic transition is characterized by an intermediate zone between the cervical lordosis and thoracic kyphosis, which leads to the transfer of load to the posterior arc. ${ }^{6}$

Anatomical consideration of cervicothoracic junction makes an anterior approach difficult due to bony structures including the sternum, clavicles and ribs in the front and large vessels, thoracic duct, and important nerves nearby; also, making the procedure fraught with high risks. The patient suffering from cervicothoracic tuberculosis due to bony structure destruction is usually complicated with regional kyphosis, which presents a further challenge to the surgeons. ${ }^{7}$ As a result, for patients with cervicothoracic tuberculosis, it is essential to individualize specific surgical approaches, based on the location of lesions to obtain optimal exposure and maximize safety for the patients.

Biomechanical studies have demonstrated that anterior plating is inferior to posterior instrumentation, and anterior instrumentation spanning over two or more levels requires additional posterior fixation in this region. ${ }^{8,9}$ Because of the anatomical consideration and radiological difficulties, posterior instrumentation is also difficult at the cervicothoracic junction.

Due to acute transition of cervicothoracic transition and variation of interpedicular distance and inner pedicular diameter, it is difficult to insert the pedicle screw in cervicothoracic junction. But recent technical advances and biomechanical studies make posterior instrumentation easier, safer and more effective. ${ }^{10}$

Various combinations are available: (1) $3.5 \mathrm{~mm}$ cervical lateral mass screws can be extended into the thoracic spine; (2) $3.5 \mathrm{~mm}$ cervical screws can be connected to the $5.5 \mathrm{~mm}$ thoracic pedicle screws by using two differing diameter rods, connected by a fixed or a hinged domino connector (wedding band); and (3) screws can be connected by tapered rods or dual diameter rods as described earlier.

The solid domino connector is an attractive option. We used a domino connector for posterior cervicothoracic fixation which provided a better construct and balance in the cervicothoracic junction. The main aim is to prevent the 

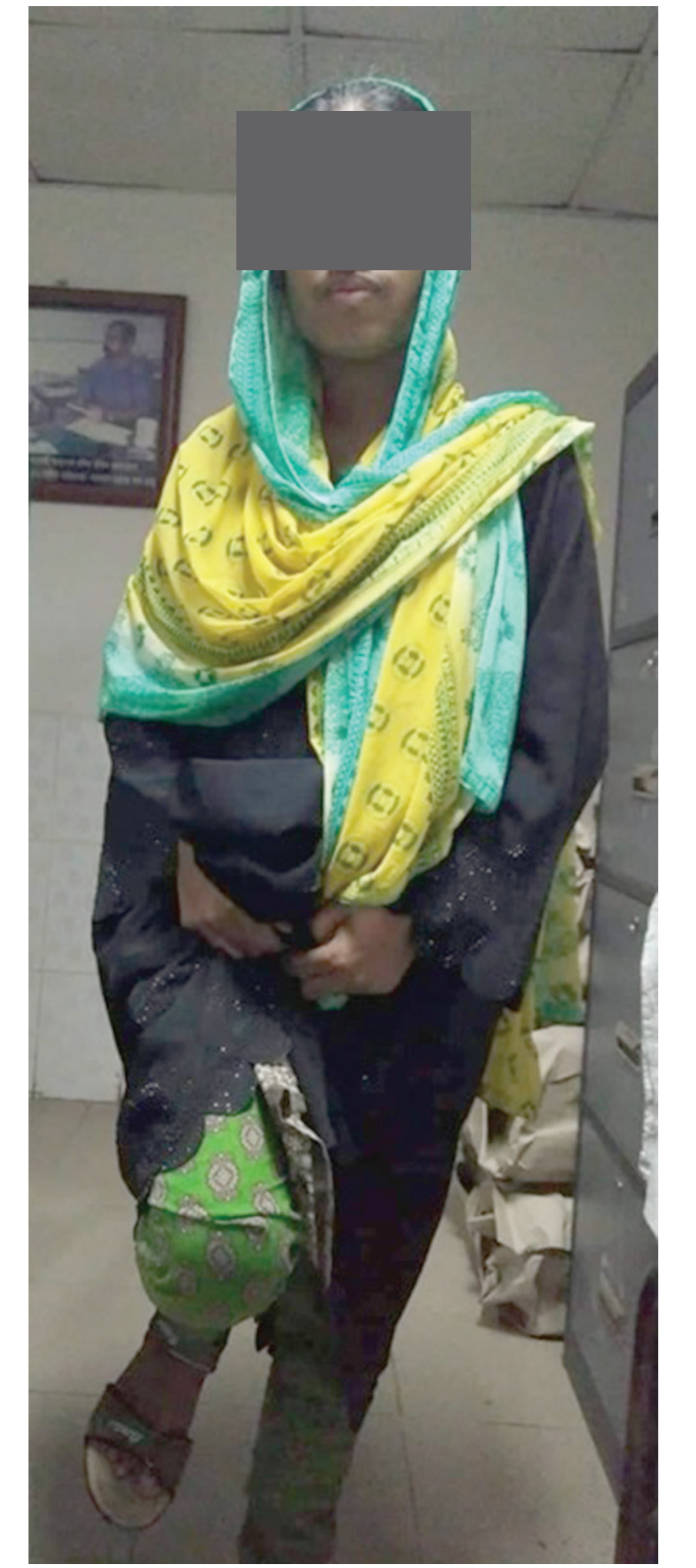

Fig. 3 After one month of operation, patient can walk independently.

further damage of neural structure and stabilize with the help of sound arthrodesis. Clinically, the patient improves significantly, indicating adequate stabilization.

\section{Conclusion}

Posterior cervicothoracic fixation in cervicothoracic junction tuberculosis with domino connector provides a very good stabilization and balance in the transitional zone of cervicothoracic junction. Further, a full understanding of the advantages and disadvantages of the potential instrumentation systems will help in avoiding unanticipated instrumentation limitations and prolonged operative times. We gave antituberculosis therapy to this patient for 18 months.

\section{Funding \\ None.}

\section{Conflict of Interest}

None declared.

\section{References}

1 Cohen ZR, Fourney DR, Gokaslan ZL, Walsh GL, Rhines LD. Anterior stabilization of the upper thoracic spine via an "interaortocaval subinnominate window": case report and description of operative technique. J Spinal Disord Tech 2004;17(6):543-548

2 Kaya RA, Türkmenoğlu ON, Koç ON, et al. A perspective for the selection of surgical approaches in patients with upper thoracic and cervicothoracic junction instabilities. Surg Neurol 2006;65(5):454-463, discussion 463

3 Jain AK. Tuberculosis of the spine: a fresh look at an old disease. J Bone Joint Surg Br 2010;92(7):905-913

4 Garg RK, Somvanshi DS. Spinal tuberculosis: a review. J Spinal Cord Med 2011;34(5):440-454

5 Zhang HQ Hu X, Yin X, Chen Y. One-stage combined anterior-posterior approach treatment of multiple cervicothoracic spinal tuberculosis with kyphosis. Int Orthop 2015;39(8):1605-1610

6 Ebraheim NA, Xu R, Knight T. Yeasting RA: morphometric evaluation of lower cervical pedicle and its projection. Spine 1997;22(1):1-6

7 Zhu Z, Hao D, Wang B, et al. Selection of surgical treatment approaches for cervicothoracic spinal tuberculosis: A 10-year case review. PLoS One 2018;13(2):e0192581

8 Korovessis P, Katonis P, Aligizakis A, et al. Posterior compact Cotrel-Dubousset instrumentation for occipitocervical, cervical and cervicothoracic fusion. Eur Spine J 2001;10(5):385-394

9 McAfee PC, Bohlman HH, Ducker TB, Zeidman SM, Goldstein JA. One-stage anterior cervical decompression and posterior stabilization. A study of one hundred patients with a minimum of two years of follow-up. J Bone Joint Surg Am 1995;77(12):1791-1800

10 Le H, Balabhadra R, Park J, Kim D. Surgical treatment of tumors involving the cervicothoracic junction. Neurosurg Focus 2003;15(5):E3 